\title{
A prospective comparison of echocardiographic wall motion score index and radionuclide ejection fraction in predicting outcome following acute myocardial infarction
}

\author{
G I W Galasko, S Basu, A Lahiri, R Senior
}

\begin{abstract}
Objective-To characterise echocardiographic wall motion score index (WMSI) as a surrogate measure of left ventricular ejection fraction (EF) following acute myocardial infarction (AMI) and to compare its prognostic value with that of EF measured by radionuclide ventriculography (RNV).

Design-A prospective study to compare baseline echocardiographic WMSI with RNV EF in consecutive patients thrombolysed for AMI, both performed on the same day before discharge, and their relative prognostic values in predicting cardiac events.

Setting-District general hospital coronary care unit and cardiology department.

Patients-120 consecutive patients free of exclusion criteria thrombolysed for AMI and followed up for a mean (SD) of 13 (10) months.

Interventions-None.

Main outcome measures-Correlation coefficients and receiver operating characteristic curve analyses plus cardiac event rates at follow up between RNV EF and echocardiographic WMSI.

Results-WMSI correlated well with RNV EF. The best corresponding WMSIs for EFs 45\%, $40 \%$, and $35 \%$ were $0.6,0.8$, and 1.1 , respectively. There were 42 cardiac events during follow up. Although both RNV EF and WMSI were strong univariate predictors of cardiac events, only WMSI independently predicted outcome in a multivariate model. All three WMSI cut offs significantly predicted events, while an RNV EF cut off of $\leqslant 45 \% v>45 \%$ failed to reach significance.

Conclusions-Although both RNV and echocardiographic WMSI strongly predicted cardiac outcome, WMSI, a cheaper and more readily available technique, is more discriminatory, especially in cases of mild left ventricular dysfunction following AMI.

(Heart 2001;86:271-276)
\end{abstract}

Keywords: echocardiographic wall motion score index; radionuclide ventriculography; prognosis; acute myocardial infarction

The assessment of left ventricular (LV) function following acute myocardial infarction (AMI) has been shown in many studies to give important prognostic information as well as helping to guide therapeutic intervention. ${ }^{1-9}$ Therapeutic studies have used global ejection fraction $(\mathrm{EF})$ as assessed by either radionuclide ventriculography (RNV) LVEF alone ${ }^{4}$ or a combination of any of RNV, echocardiographic LVEF, and ventricular angiographic $\mathrm{LVEF}^{37}$ as markers for LV dysfunction. More recently studies have used echocardiographic wall motion score index (WSMI) ${ }^{8}$ as a marker for LV dysfunction. However, none has directly compared one method with another in a prospective manner in determining prognosis, assuming each technique to be equivalent. Recent retrospective analyses of those studies where more than one method for determining LVEF was used have suggested that these measures of LV function may not be equivalent. $^{1011}$

Echocardiographic WMSI is a relatively easily obtained marker of global LV dysfunction after myocardial infarction. ${ }^{12}{ }^{13}$ It is derived by grading the wall motion of individual myocardial segments and dividing the total score by the number of analysable segments. Although several studies have shown that WMSI correlates well with LVEF determined by RNV, ${ }^{12}{ }^{14}$ 16 no studies thus far have analysed what WMSI best corresponds to LVEF in their subjects before discriminating outcome, and none has directly compared WMSI with LVEF in the assessment of prognosis.

The purpose of our study, therefore, was to characterise postmyocardial infarction echocardiographic WMSI as a measure of EF both in the immediate aftermath of acute myocardial infarction (AMI) and at six month follow up, by using RNV as a traditional measure of LVEF, ${ }^{17-20}$ and to compare their relative prognostic values when performed at the time of hospital discharge following AMI. To minimise confounding clinical factors, only patients with clinically uncomplicated AMI at the time of hospital discharge were selected.

\section{Methods}

SUBJECTS

This was a prospective study of 147 consecutive patients treated with thrombolysis following AMI (123 men, 24 women, 73 anterior territory and 74 inferior territory infarctions). 
Myocardial infarction was diagnosed on the basis of a combination of typical anginal pain of at least 30 minutes in duration, diagnostic serial electrocardiographic changes consisting of new pathological $\mathrm{Q}$ waves or ST and $\mathrm{T}$ wave changes, and a typical rise and fall in the concentration of serum total creatinine kinase or creatine kinase-MB. One hundred and twenty two patients (103 men, 19 women) were assessed by both echocardiography and RNV on the same day before discharge within 5-8 days of myocardial infarction. Eighteen were excluded before predischarge investigation for the following reasons: three died, three had another infarction, six developed unstable angina, three developed severe congestive heart failure, two developed intractable arrhythmias, and one developed poorly controlled hypertension. Seven others were excluded for logistical reasons with either echocardiography or RNV being unable to be performed before discharge. A WMSI could not be obtained in two of the 122 patients studied predischarge due to inadequate image quality. Eighty six patients went on to undergo echocardiography and RNV at six months' follow up, with a WMSI unable to be performed in one patient because of inadequate image quality.

RADIONUCLIDE VENTRICULOGRAPHY

Red blood cells were labelled in vivo by an intravenous injection of $10 \mathrm{mg}$ stannous pyrophosphate followed by an injection of $740 \mathrm{MBq}$ of technetium ${ }^{99 \mathrm{~m}} 30$ minutes later. Multiple gated RNVs were obtained by means of a gamma camera using a low energy collimator (Elscint, Apex 215M, Haifa, Israel). The camera was positioned in the $30-45^{\circ}$ left anterior oblique projection with a $5-10^{\circ}$ caudal tilt to isolate the $\mathrm{LV}$. The $\mathrm{R}-\mathrm{R}$ interval was divided into 32 frames and a 5\% gate tolerance was used. Five million counts were collected and the data stored in a $64 \times 64$ matrix to obtain a high resolution time-activity curve of the change in precordial radioactivity. The LVEF was obtained in the left anterior oblique view using a second derivative semiautomatic edge detection programme, as previously described by our laboratory, ${ }^{21}$ and reported by one observer (AL). We have previously published the interobserver and intraobserver variability in measuring RNV EF following AMI in our laboratory in 24 and 21 patients, respectively, finding no significant overall mean differences and limits of agreement of -9.4 to $7.6 \%$ and -8.6 to $11 \%$, respectively. ${ }^{19}$

\section{TWO DIMENSIONAL ECHOCARDIOGRAPHY}

Two dimensional echocardiography was performed by one investigator (RS) using a phased array ultrasound imaging system with a $2.25 \mathrm{MHz}$ transducer (ATL, Ultramark 9, Bothell, Washington, USA). Parasternal long and short axis and apical four and two chamber views were obtained. WMSI was analysed using an 11 segment model (basal lateral, middle lateral, basal inferior, middle inferior, basal posterior interventricular septum, middle posterior interventricular septum, basal anterior free wall, middle anterior free wall, basal anterior interventricular septum, middle anterior interventricular septum and apex) with six segments each assigned to anterior and inferior regions, the apex being common. The motion of individual segments was graded as follows: normal 0 , hypokinesia 1 , akinesia 2 , and dyskinesia 3. Global systolic wall motion score was calculated by dividing the total score by the number of segments analysable. Results were only included when at least four segments from each of the anterior and inferior regions were analysable. We have previously published the intra-observer variability of WMSI in 50 subjects post-AMI in our laboratory finding a concordance of $91 \%$ with a kappa value of $0.78 .^{22}$

FOLLOW UP

All patients were reassessed by formal review for first cardiac events. The cardiac end points were death, reinfarction, and hospitalisation for either unstable angina, congestive heart failure, or life threatening arrhythmia. Revascularisation alone was not considered a cardiac end point. Unstable angina was defined as new onset or worsening of previous angina requiring hospital admission with electrocardiographic evidence of ischaemia. Congestive heart failure was defined as new onset of clinical heart failure or worsening of heart failure according to the New York Heart Association classification requiring hospitalisation accompanied by basal rales, third heart sound, or evidence of pulmonary upper lobe diversion on chest radiography.

\section{STATISTICAL ANALYSIS}

The correlation coefficient between RNV EF and WMSI was calculated for predischarge and six month follow up studies and in both combined. Receiver operating characteristic curves were constructed for the ability of WMSI measurements to predict specific prechosen abnormal RNV EF ranges. These prechosen clinically significant EFs were $\leqslant 45 \%, \leqslant 40 \%$, and $\leqslant 35 \%$. The predischarge WMSI with the highest accuracy and $\kappa$ at each of these EFs was calculated by receiver operating characteristic curves. Linear regression analysis for WMSI to predict LVEF was also performed. $\kappa<0.40$ was considered poor agreement, $0.40-0.59$ moderate, 0.600.79 good, and $\geqslant 0.80$ excellent agreement. The area under the receiver operating characteristic curves was calculated to give the overall accuracy of WMSI in predicting a given $\mathrm{EF}^{23}$

A univariate Cox proportional hazards regression model was created for demographic and clinical variables including WMSI and RNV EF to predict cardiac events. Stepwise Cox regression analysis was then performed in a forward stepwise manner until all variables remaining in the multivariate model were significant at the $5 \%$ level to find independently predictive variables. Time to event curves were generated using Kaplan-Meier estimates, with the log rank test used for equality of event-free survival analysis. Prognostic groups analysed were RNV EFs $\leqslant 45 \%$ v > $45 \%$, 

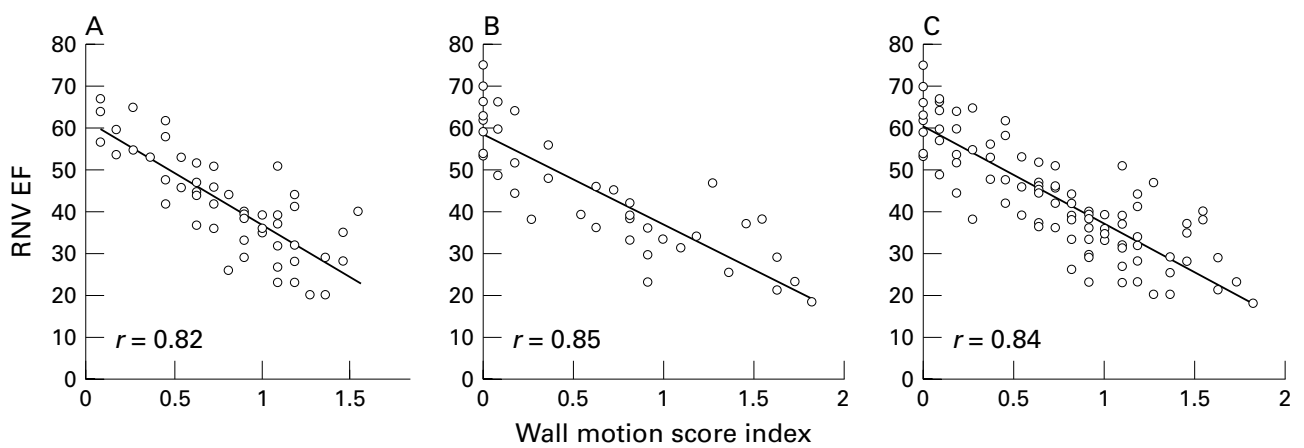

Figure 1 Scatter plot of radionuclide ventriculographic ejection fraction (RNV EF) compared with echocardiographic wall motion score index (WMSI) (A) in 120 patients pre-discharge after myocardial infarction, (B) in 85 patients six months after myocardial infarction, and $(C)$ in all 205 patients combined.

$\leqslant 40 \% v>40 \%$, and $\leqslant 35 \% v>35 \%$, along with the most comparable WMSI from the receiver operating characteristic analyses. All continuous variables are expressed as mean (1SD) and all categorical variables as proportions or percentages. Data were analysed using SPSS version 9.0 (SPSS Inc, Chicago, Illinois, USA).

\section{Results}

PATIENTS

The mean (SD) age of the 120 patients fully assessed before discharge was 60.4 (11.1) years (range 34-83 years); 70 were white, 48 were south Asian, and two were African Caribbean. All patients had received thrombolysis. There were 56 anterior and 64 inferior myocardial infarctions. There were no significant differences in demographics, baseline clinical features, or drug treatments between those with abnormal RNV EFs and those with the equivalent abnormal WMSI at all three levels of EF studied.

COMPARISON OF RNV WITH WMSI

A scatter plot showing the overall correlation between RNV EF and WMSI is depicted in fig 1 , with correlation coefficients given. Correlation coefficients were 0.82 and 0.85 for anterior infarctions before discharge and at six months' follow up, respectively, and 0.65 and 0.80 for inferior infarctions before discharge and at six months' follow up, respectively.

Receiver operating characteristic curves were derived for predischarge WMSI to predict given RNV EFs (fig 2). Areas under the curve were $0.95,0.95$, and 0.94 for $\mathrm{EF} \leqslant 45 \%$,
Table 1 Agreement between radionuclide ejection fraction (EF) and echocardiographic wall motion score index (WMSI) measurements in assessing mild left ventricular dysfunction ( $E F \leqslant 45 \%$ or WMSI $\geqslant 0.6$ ) in 120 subjects before discharge following acute myocardial infarction

\begin{tabular}{llll}
\hline & \multicolumn{2}{c}{ Radionuclide $E F$} & \\
\cline { 2 - 3 } & $\leqslant 45 \%$ & $>45 \%$ & Total \\
\hline Echocardiographic WMSI & & & \\
$\quad \geqslant 0.6$ & 40 & 7 & 47 \\
$\quad<0.6$ & 5 & 68 & 73 \\
Total & 45 & 75 & 120 \\
\hline
\end{tabular}

$\leqslant 40 \%$, and $\leqslant 35 \%$, respectively. The respective WMSIs with the highest predictive accuracies for these EFs were WMSI $\geqslant 0.6$ (accuracy $90 \%, \quad \kappa=0.79$ ),$\geqslant 0.8 \quad$ (accuracy 93\%, $\kappa=0.81$ ), and $\geqslant 1.1 \quad$ (accuracy $89 \%$, $\kappa=0.60)$. The agreement between RNV EF and WMSI measurements in subjects with mild LV dysfunction (EF $\leqslant 45 \%$ or WMSI $\geqslant 0.6)$ is shown in table 1 . Corresponding WMSI scores from the predischarge linear regression plots were 0.7 (accuracy 89\%, $\kappa=0.76$ ), 0.9 (accuracy $92 \%, \kappa=0.78$ ), and 1.1 (accuracy 89\%, $\kappa=0.60$ ), respectively. Receiver operating characteristic curves and linear regression analyses were also calculated for all 205 scans combined, finding areas under the curve of 0.95 throughout.

The mean (SD) RNV EF in anterior myocardial infarctions was $42.4 \quad(12.3) \%$ (range 20-67) with an $\mathrm{EF} \leqslant 45 \%$ found in 34 of the 56 patients $(61 \%)$. The mean (SD) RNV $\mathrm{EF}$ in inferior myocardial infarctions was 54.6 (9.6) \% (range 31-74) with an $\mathrm{EF} \leqslant 45 \%$ found in 11 of the 64 patients $(17 \%)$. The mean (SD) WMSI in anterior myocardial
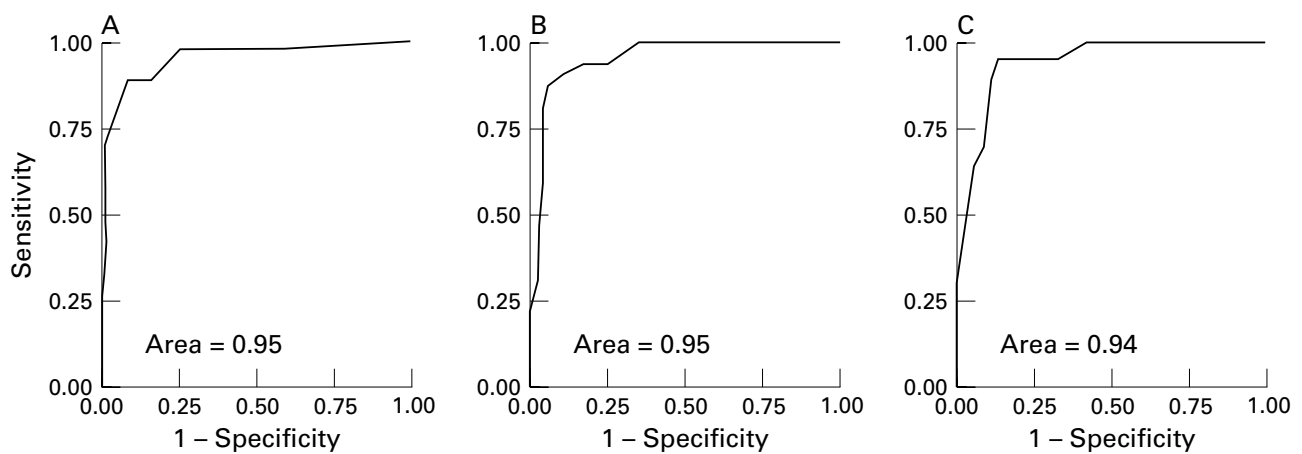

Figure 2 Receiver operating characteristic curves for the pre-discharge echocardiographic WMSI to predict $(A)$ an RNV $E F$ of $\leqslant 45 \%$, (B) an $R N V E F$ of $\leqslant 40 \%$, and (C) an $R N V E F$ of $\leqslant 35 \%$. 


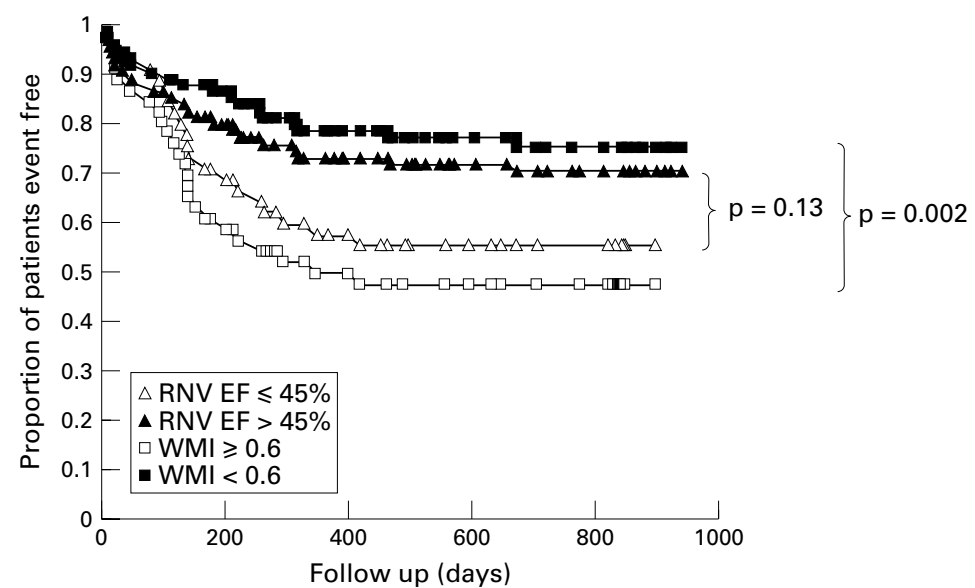

Figure 3 Kaplan-Meier event free survival curves comparing cardiac event free survival for radionuclide ejection fraction (RNV EF) $\leqslant 45 \%$ versus $>45 \%$ and echocardiographic wall motion score index (WMSI) $\geqslant 0.6$ versus $<0.6$ in 120 patients pre-discharge following acute myocardial infarction.

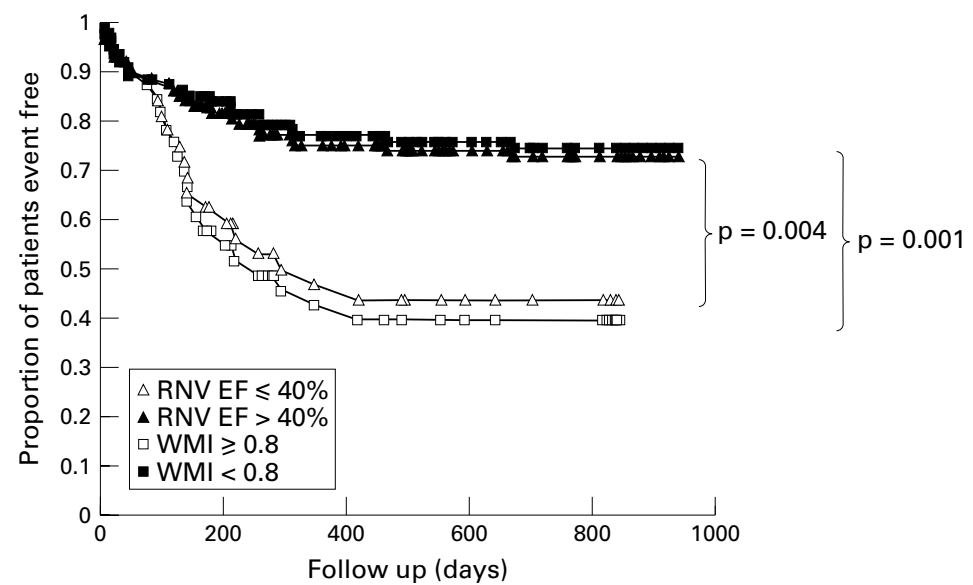

Figure 4 Kaplan-Meier event free survival curves comparing cardiac event free survival for $R N V E F \leqslant 40 \%$ versus $>40 \%$ and echocardiographic WMSI $\geqslant 0.8 v<0.8$ in 120 patients pre-discharge following acute myocardial infarction.

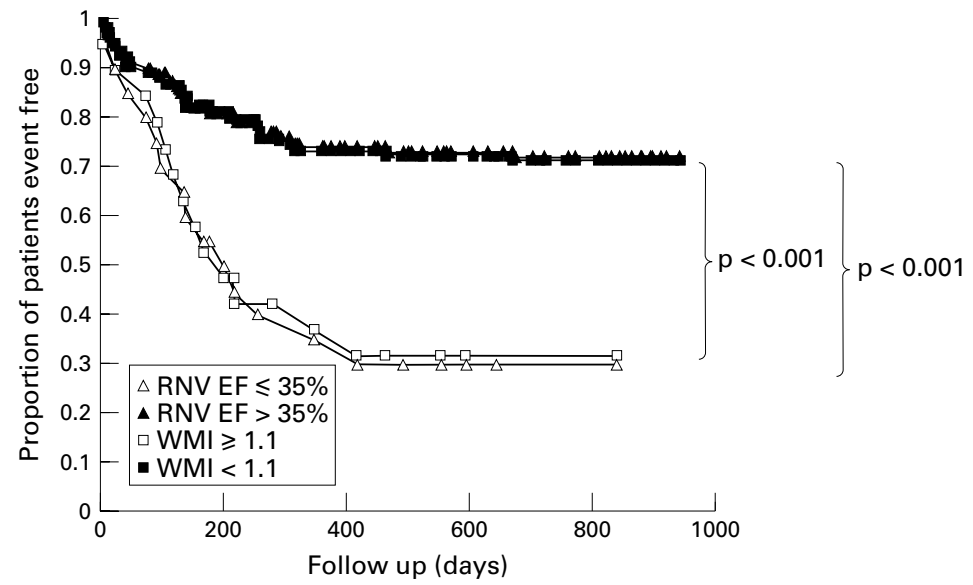

Figure 5 Kaplan-Meier event free survival curves comparing cardiac event free survival for $R N V E F \leqslant 35 \%$ versus $>35 \%$ and echocardiographic WMSI $\geqslant 1.1$ versus $<1.1$ in 120 patients pre-discharge following acute myocardial infarction.

infarctions was $0.78(0.40)$ (range $0.1-1.5$ ) with a WMSI $\geqslant 0.6$ in 38 of the 56 patients $(68 \%)$. The mean (SD) WMSI in inferior myocardial infarctions was $0.37(0.24)$ (range $0-1.4)$ with a WMSI $\geqslant 0.6$ in 9 of the 64 patients $(14 \%)$.
PROGNOSIS

Patients were followed up for first cardiac events for a mean of 13 (10) months (range 5-942 days). During this time there were 42 first cardiac events ( 3 deaths, 8 reinfarctions, 18 cases of unstable angina requiring hospitalisation, and 13 episodes of new or worsening congestive heart failure requiring hospitalisation). There were no cases of life threatening arrhythmia requiring hospitalisation. Four patients went on to require elective coronary artery bypass surgery and three to angioplasty. None of these seven patients, however, developed cardiac events during follow up.

Cox regression analyses

Univariate Cox regression analysis showed that WMSI $\left(\chi^{2}=13.0, \mathrm{p}=0.0003\right), \mathrm{RNV} \mathrm{EF}$ $\left(\chi^{2}=7.9, \mathrm{p}=0.005\right)$, ethnic origin $\left(\chi^{2}=4.4\right.$, $\mathrm{p}=0.04)$, and age $\left(\chi^{2}=4.4, \mathrm{p}=0.04\right)$ all significantly predicted cardiac events. When multivariate analysis was performed, WMSI $\left(\chi^{2}=11.9, \mathrm{p}=0.006\right)$, ethnic origin $\left(\chi^{2}=6.1\right.$, $\mathrm{p}=0.01)$, and age $\left(\chi^{2}=5.3, \mathrm{p}=0.02\right)$ were the only independent predictors of cardiac events, with RNV EF no longer predicting outcome.

Event free survival analysis

Cumulative event free survival was assessed for a range of EFs felt to be of clinical significance and their corresponding WMSI with greatest agreement ( $\mathrm{EF} \leqslant 45 \% \quad v>45 \%$ compared with WMSI $<0.6 v \geqslant 0.6$; EF $\leqslant 40 \% v>40 \%$ compared with WMSI $<0.8 v \geqslant 0.8$; and EF $\leqslant 35 \% v>35 \%$ compared with WMSI of $<1.1 v \geqslant 1.1$ ).

Nine hundred and forty two day KaplanMeier cumulative event-free survival curves are shown for the various cut offs of RNV EF and their corresponding WMSI (figs 3, 4, and 5). WMSIs significantly predicted survival for all cut offs, while radionuclide derived EF failed to reach significance at a cut off of $45 \%$ $(p=0.13)$. At this cut off, the relative risk of a cardiac event in the RNV EF group compared with the WMSI group was 0.85 (95\% confidence interval 0.56 to 1.3 ) ( $\mathrm{p}=0.38, \log$ rank test).

Five of the 45 patients with a clinically significant reduction in RNV EF ( $\leqslant 45 \%)$ did not have a clinically significant wall motion abnormality (WMSI < 0.6). Of these, only one had a cardiac event during follow up. Of the 75 patients with an RNV EF of $>45 \%$, seven had a clinically significant wall motion abnormality (WMSI $\geqslant 0.6$ ). Of these, five had cardiac events during follow up.

\section{Discussion}

This study has provided further evidence that WMSI is an easily measurable marker of LV function following AMI, being obtainable in 205 of 208 echocardiographic studies performed $(98.6 \%)$ even in the absence of second harmonic imaging, ${ }^{24}$ as described elsewhere. ${ }^{12}{ }^{13}$ It has also confirmed the very close correlation between WMSI and RNV EF both acutely and chronically post AMI, also described elsewhere. ${ }^{12}{ }^{16}$ This study has further 
found a closer correlation for anterior infarctions than inferior infarctions, especially before discharge.

It has also shown a very good agreement between WMSI and RNV EF with areas under the receiver operating characteristic curve ranging from 0.94 to 0.95 at the clinically significant cut offs in $\mathrm{EF}$ of $45 \%, 40 \%$, and $35 \%$. This implies a $94-95 \%$ probability that two subjects, one with a clinically significant abnormality in RNV EF and one without, would be correctly discriminated by an echocardiographic WMSI. ${ }^{23}$

We found that WMSIs of $0.6,0.8$, and 1.1 corresponded best to EFs $45 \%, 40 \%$, and $35 \%$, respectively. This differed slightly from the linear regression plot where the corresponding WMSIs were $0.7,0.9$, and 1.1 , respectively, suggesting a non-linear relation between WMSI and RNV EF. The only previous study that analysed the relation between RNV EF and echocardiographic WMSI ${ }^{16}$ also found a non-linear relation, with their linear regression analysis producing an overestimation of low EFs and an underestimation of high EFs. Our data suggest that a greater degree of wall motion abnormality must take place before a lower EF occurs.

PROGNOSIS

Several studies have shown LV systolic dysfunction to be one of the most important risk factors for morbidity and mortality following AMI, whether assessed by WMSI or by global $\mathrm{EF}$, and whether assessed by echocardiography or by RNV. ${ }^{1}{ }^{1325-29}$ This study further supports this, with both RNV EF and WMSI strong univariate predictors of cardiac events. However, in this first direct comparison, only echocardiographic WMSI independently predicted adverse events in a multivariate model. A direct comparison at EFs ranging from mild to more severe LV dysfunction suggested that these differences may be most significant in patients with mild LV dysfunction, with a relative risk of cardiac events of 0.85 for RNV EF $\leqslant 45 \%$ compared with the equivalent echocardiographic WMSI $\geqslant 0.6$.

One plausible explanation for increased discrimination of WMSI over RNV EF at mild LV dysfunction may be the commonly observed phenomenon of compensatory hyperkinesis of non-involved myocardium. ${ }^{30}$ This would limit the reduction in global $\mathrm{EF}$ resulting from akinesis of the infarcted muscle while not affecting the WMSI, with WMSI acting as a more precise measure of myocardial damage. In more extensive infarctions with more myocardial involvement, there is less scope for this compensatory hyperkinesis, with both WMSI and RNV EF accurately reflecting myocardial damage, giving similar prognostic information. This may explain our finding that WMSI correlates relatively poorly with RNV EF before discharge after inferior myocardial infarction when compensatory anterior hyperkinesia may mask the degree of myocardial damage as compared with at six months after infarction when compensatory hyperkinesia no longer occurs. Another possible mechanism may include methodological errors in calculating radionuclide $\mathrm{EF}$, with this technique relying on total ventricular counts and so allowing an excessive contribution to the overall counts from the anterior ventricular cavity as it lies nearer the chest wall and thus nearer the gamma camera. ${ }^{11}{ }^{31}$ Thus, patients with large akinetic anterolateral walls or apices may have a greater contribution to overall counts from these diseased areas, underestimating EF, while patients with inferoposterior infarctions may give a reduced contribution to overall counts from affected regions, overestimating the EF. Since the majority of patients with anterior myocardial infarctions had an $\mathrm{EF}$ $\leqslant 45 \%$ by RNV EF and the majority with inferior myocardial infarctions an $\mathrm{EF}>45 \%$, underestimating the EF in anterior myocardial infarctions and overestimating the $\mathrm{EF}$ in inferior myocardial infarctions would reduce discrimination in cases of mild LV dysfunction.

Although this is the first study to compare outcome by echocardiographic WMSI compared with EF by RNV after myocardial infarction, comparisons between echocardiographic EF and RNV EF also found differences in prognosis between echocardiography and RNV. A retrospective analysis of the SOLVD (studies of left ventricular dysfunction) database found that an echocardiographic EF $\leqslant 35 \%$ was more discriminatory for both overall death and cardiovascular mortality than an RNV EF $\leqslant 35 \%$ following $\mathrm{AMI},{ }^{10}$ no data being given at higher EF cut offs. Similarly, Barbir and colleagues ${ }^{32}$ found that an echocardiographic $\mathrm{EF}<60 \%$, but not a RNV EF $<60 \%$, independently predicted cardiovascular events in 18 heart transplant recipients over a 2.1 year follow up.

\section{STUDY LIMITATIONS}

The present study has a number of limitations. Firstly, it was a relatively small study of 120 patients, so that although multivariate analysis found WMSI to be more discriminatory for cardiac events than RNV EF, the numbers were too small to show definitively at what level of LV dysfunction this difference is most apparent. Secondly, this study took place in one centre with echocardiography performed by one operator and RNV EF by one operator and so requires confirmation elsewhere. Thirdly, although RNV is a well validated semiquantitative technique, echocardiographic wall motion assessment is a qualitative technique, previously shown to give only fair interobserver variability when endocardial definition is less than perfect. ${ }^{33}$ However, this same study showed $100 \%$ agreement in the few cases with complete endocardial definition, nowadays more easily achieved by use of tissue harmonic imaging ${ }^{24}$ and contrast agents. ${ }^{34}$ Agreement can be further improved by use of quantitative techniques that are under investigation including segmental analysis of colour kinetic images ${ }^{35}$ and tissue Doppler imaging, ${ }^{36}$ potentially quantifying regional $\mathrm{LV}$ function and thus WMSI. Finally, again because of the small number of patients enrolled, the cardiac events discussed may be described as "soft" 
rather than hard, although clearly of great clinical significance. However, it is the first direct comparison between these two previously thought to be equivalent techniques, ${ }^{8}$ techniques used to assess LV function both in clinical practice and in large clinical trials, finding differing results in a prospective manner.

CONCLUSION

Echocardiographic WMSI may be performed successfully in the vast majority of patients following AMI and is an accurate marker of LV dysfunction. Furthermore, it gives important prognostic information in the postmyocardial infarction setting, more so than RNV EF, a well established and previously thought to be equivalent technique, with this difference in prognostic power appearing to be most dramatic in cases of mild LV dysfunction, although larger studies are needed to show this definitively. Further advantages may include cost, availability, absence of exposure to radiation, and the ability to assess common potential complications of AMIs such as valvar dysfunction or ventricular septal defects.

This study was funded by the Northwick Park Hospital Cardiac Research Fund. We would like to thank Christopher Kinsey and Usha Raval for their technical assistance.

1 Heger JJ, Weyman AE, Wann LS, et al. Cross-sectional echocardiographic analysis of the left ventricular asynergy

2 Kan G, Visser CA, Koolen JJ, et al. Short and long term predictive value of admission wall motion score in acute myodictive value of admission wall motion score in acute myo-
cardial infarction. A cross sectional echocardiographic cardial infarction. A cross sectional echocardit
study of 345 patients. Br Heart $\mathcal{~ 1 9 8 6 ; 5 6 : 4 2 2 - 7 . ~}$

3 The SOLVD Investigators. Effect of enalapril on survival in patients with reduced left ventricular ejection fraction and patients with reduced left ventricular ejection fraction and

4 Pfeffer MA, Braunwald E, Moye L. Effect of captopril on mortality and morbidity in patients with left ventricular dysfunction after myocardial infarction. $N$ Engl $\mathcal{F} \mathrm{Med}$ 1992;327:669-77

5 Volpi A, De Vita C, Franzosi MG, et al. Determinants of 5 -month mortality in survivors of myocardial infarction after thrombolysis, results of the GISSI-2 data base. Circulation 1993;88:416-29.

6 The Acute Infarction Ramipril Efficacy (AIRE) study investigators. Effect of ramipril on mortality and morbidity of survivors of acute myocardial infarction with clinical survivors of acute myocardial infarction with

7 Waldo AL, Camm AJ, deRuyter H, et al. Survival with oral Waldo AL, Camm AJ, deRuyter H, et al. Survival with oral d-sotalol in patients with left ventricular dysfunction after
myocardial infarction: rationale, design, and methods (the myocardial infarction: rationale, design, and m.

8 Kober L, Torp-Pedersen C, Carlsen JE, et al. A clinical trial of the angiotensin-converting enzyme inhibitor trandolapril in patients with left ventricular dysfunction after myocar dial infarction. N Engl F Med 1995;333:1670-6.

9 The DIAMOND study group. Dofetilide in patients with left ventricular dysfunction and either heart failure or acute myocardial infarction: rationale, design, and patien characteristics of the DIAMOND studies. Clin Cardio 1997;20:704-10.

10 Rashid H, Exner DV, Mirsky I, et al. Comparison of echocardiography and radionuclide angiography as predictors of mortality in patients with left ventricular dysfunction (studies of left ventricular dysfunction). Am $\mathcal{f}$ Cardiol 1999;84:299-303.

11 Urena PE, Gervasio AL, Mitchell G, et al. Ejection fraction by radionuclide ventriculography and contrast left by radionuclide ventriculography and contrast left
ventriculography: a tale of two techniques. $\mathcal{f} \mathrm{Am}$ Coll ventriculography: a tale

12 Jensen-Urstad K, Bouvier F, Hojer J, et al. Comparison of different echocardiographic methods with radionuclide imaging for measuring left ventricular ejection fraction during acute myocardial infarction treated by thrombolytic therapy. Am $\mathcal{F}$ Cardiol 1998;81:538-44.

13 Berning J, Steensgaard-Hansen F. Early estimation of risk by echocardiographic determination of wall motion index in an unselected population with acute myocardial infarction. Am $\mathcal{7}$ Cardiol 1990;65:567-76.

14 Rifkin RD, Koito H, Farmelant M, et al. Segmental model for estimating left ventricular ejection fraction by twodimensional echocardiography: comparison with gated blood pool scanning. Am F Cardiol 1990;4:193-202.

15 Rifkin $\mathrm{RD}$, Koito $\mathrm{H}$. Comparison with radionuclide angiography of two new geometric and four non geometric models for echocardiographic estimation of left ventricular ejection fraction using segmental wall motion scoring. Am 7 Cardiol 1990;65:1485-90.

16 Berning J, Rokkedal-Nielson J, Launbjerg J, et al. Rapid estimation of left ventricular ejection fraction in acute myocardial infarction by echocardiographic wall motion analysis. Cardiology 1992;80:257-66.

17 Wackers FJ, Berger HJ, Johnstone DE, et al. Multiple gated cardiac blood pool imaging for EF: validation of the technique and assessment of variability. Circulation 1979; 43:1159-66.

18 Upton MT, Rerych SK, Newman GE, et al. The reproducibility of radionuclide angiographic measurements of left ventricular function in normal subjects at rest and during exercise. Circulation 1980;62:126-32.

19 Senior R, Sridhara BS, Basu S, et al. Comparison of radionuclide ventriculography and $2 \mathrm{D}$ echocardiography for the measurement of left ventricular ejection fraction following acute myocardial infarction. Eur Heart f 1994;15:1235-9.

20 Van Royen N, Jaffe CC, Krumholz HM, et al. Comparison and reproducibility of visual echocardiographic and quantitative radionuclide left ventricular ejection fractions. $A m \mathcal{F}$ Cardiol 1996;77:843-50.

21 Haines AD, Khawaja IA, Hinge DA, et al. Radionuclide left ventricular ejection fraction: a comparison of three methods. Br Heart f 1987;57:242-6.

22 Senior R, Basu S, Khattar R, et al. Independent prognostic value of the extent and severity of systolic wall thickening abnormality at infarct site after thrombolytic therapy. Am Heart 7 1998;135:1093-8.

23 Hanley JA, McNeill BJ. The meaning and use of the area under a receiver operator characteristic (ROC) curve. Radiology 1982;143:29-36.

24 Senior R, Soman P, Khattar RS, et al. Improved endocardial visualization with second harmonic imaging compared with fundamental two-dimensional echocardiographic imaging. Am Heart F 1999;138:163-8

25 Kelly MJ, Thompson PL, Quinlan MF. Prognostic significance of left ventricular ejection fraction after acute myocardial infarction. A bedside radionuclide study. $\mathrm{Br}$ Heart f 1985;53:16-24.

26 Ong L, Green S, Reiser P, et al. Early prediction of mortality in patients with acute myocardial infarction: a prospective study of clinical and radionuclide risk factors. Am $\mathcal{F}$ Cardiol 1986;57:33-8.

27 Dwyer EM, Greenstein HM, Steinberg G and the Multicenter Postinfarction research group. Clinical characteristics and natural history of survivors of pulmonary congestion during acute myocardial infarction. Am $\mathcal{f}$ Cardiol

28 Kober L, Trop-Pederson C. Clinical characteristics and mortality of patients screened for entry into the trandolapril cardiac evaluation (TRACE) study. Am $f$ Cardiol 1995;76:1-5.

29 Volpi A, De Vita C, Franzosi MG, et al. Determinants of 6-month mortality in survivors of myocardial infarction after thrombolysis. Results of the GISSI-2 database. Circulation 1993;88:416-29.

30 Kjoller E, Kober L, Jorgensen S, et al. Long-term prognostic importance of hyperkinesias following acute myocardial
infarction. TRACE study group. Trandolapril cardiac evaluation. Am $\mathcal{F}$ Cardiol 1999;83:655-9.

31 Schneider RM, Jaszczak RJ, Coleman RE, et al. Disproportionate effects of regional hypokinesis on radionuclide ejection fraction: compensation using attenuation-corrected tion fraction: compensation using attenuation-cor
ventricular volumes. $\mathcal{F ~} \mathrm{Nucl}$ Med 1984;25:747-54.

32 Barbir M, Lazem F, Banner N, et al. The prognostic significance of non-invasive cardiac tests in heart transplant recipients. Eur Heart $\mathcal{F}$ 1997;18:692-6.

33 Hoffman R, Lethen H, Marwick T, et al. Analysis of interinstitutional observer agreement in interpretation of dobutamine stress echocardiograms. If Am Coll Cardiol 1996;27:330-6

34 Senior R, Andersson O, Caidahl K, et al. Enhanced left ventricular endocardial border delineation with an intravenous injection of sonuvue, a new echocardiographic contrast agent: a European multicentre study. Echocardiography 2000;17:705-11.

35 Koch R, Lang RM, Garcia MJ, et al. Objective evaluation of regional left ventricular wall motion during dobutamine stress echocardiographic studies using segmental analysis of color kinesis images. f Am Coll Cardiol 1999;34:409-19.

36 Gorcsan J 3 ${ }^{\text {rd }}$, Strum DP, Mandarino WA, et al. Quantitative assessment of alterations in regional left ventricular contractility with color-coded tissue Doppler echocardiography. Comparison with sonomicrometry and pressurevolume relations. Circulation 1997;95:2423-33. 